FORMATLON Formation emploi

Revue française de sciences sociales

132 | octobre-décembre 2015

Enseignement supérieur et mondes économiques : de nouveaux éclairages

\title{
Introduction : Nouveaux éclairages sur les relations entre enseignement supérieur et monde économique
}

Olivia Chambard et Laurène Le Cozanet

\section{CpenEdition}

Journals

Édition électronique

URL : http://journals.openedition.org/formationemploi/4530

DOI : 10.4000/formationemploi.4530

ISSN : 2107-0946

Éditeur

La Documentation française

Édition imprimée

Date de publication : 30 décembre 2015

Pagination : 7-13

ISSN : 0759-6340

Référence électronique

Olivia Chambard et Laurène Le Cozanet, «Introduction : Nouveaux éclairages sur les relations entre enseignement supérieur et monde économique », Formation emploi [En ligne], 132 | octobre-décembre 2015, mis en ligne le 28 janvier 2016, consulté le 30 octobre 2020. URL : http://

journals.openedition.org/formationemploi/4530 ; DOI : https://doi.org/10.4000/formationemploi.4530

(c) Tous droits réservés 


\title{
Introduction
}

\section{Nouveaux éclairages sur les relations entre enseignement supérieur et monde économique}

\author{
Olivia Chambard \\ Doctorante en sciences sociales (Centre Maurice Halbwachs, Ecole des hautes études \\ en sciences sociales - Ecole normale supérieure) et professeure agrégée en sciences \\ économiques et sociales (université Paris 1 Panthéon-Sorbonne) \\ LAurène Le CozAnet \\ Doctorante en science politique (Institut de Recherche Interdisciplinaire en sciences \\ sociales, Paris Dauphine)
}

Comme l'énonçait déjà Émile Durkheim (1922), l'éducation constitue un enjeu social central, dans la mesure où chaque société possède " un certain idéal de l'homme, de ce qu'il doit être tant au point de vue intellectuel que physique et moral " et tente de former des hommes dans cette perspective. Aujourd'hui, le constat d'un capitalisme semblant pénétrer toujours plus profondément dans nos vies invite à s'interroger sur la place des institutions éducatives dans ce mouvement : quelle relation entretiennent-elles avec les dynamiques économiques ? Dans quelle mesure y contribuent-elles ? De quelle manière sont-elles travaillées, transformées par celles-ci ?

Si les études sur les relations entre éducation et système productif ont longtemps privilégié l'enseignement secondaire professionnel ${ }^{1}$, des sociologues et des économistes se sont plus précisément intéressés, ces dernières années, aux rapports entre l'enseignement supérieur et le monde économique. Ce faisant, ils les ont souvent appréhendés au prisme des questions d'insertion professionnelle ${ }^{2}$, approfondissant les interrogations soulevées de longue date par les enquêtes statistiques du Céreq. La centralité de cette thématique, dans les travaux scientifiques comme dans la construction des problèmes publics $^{3}$, dissimule toutefois la complexité de ces relations.

Celles-ci ne s'articulent pas uniquement autour d'un transfert de diplômés vers l'emploi et des conditions de sa réalisation mais aussi autour d'enjeux aussi variés que la participation du secteur privé au financement de l'enseignement supérieur, l'intervention

1. Voir par exemple : Maurice, Sellier, Sylvestre, 1982 ; Moreau, 1999 ; Ropé, Tanguy, 1994.

2. Voir par exemple : Hugrée (2010), Poullaouec (2010), Giret (2011).

3. A l'image d'un récent rapport pour le ministère de l'Education nationale, de l'Enseignement supérieur et de la Recherche, intitulé Evaluation du partenariat de l'Education nationale et de l'Enseignement supérieur avec le monde économique pour l'insertion professionnelle des jeunes (2015). 
de professionnels ${ }^{4}$ dans l'enseignement, la valorisation économique de la recherche, l'importation de méthodes de gestion du secteur privé, etc. Ces phénomènes, sans être inédits ${ }^{5}$, connaissent une nouvelle résonance à l'aube du XXIe siècle, alors que des questions telles que la poursuite de la massification des études supérieures, la persistance du chômage des jeunes diplômés, les difficultés de financement de l'enseignement supérieur ou encore la compétition académique nationale et internationale se trouvent au cœur de vifs débats dans les arènes politique, médiatique et universitaire. Notons que, dans le même temps, les discours incitant au changement ${ }^{6}$ comme les transformations effectives en termes de formation (Garcia, 2009; Chambard, 2013), de recherche (Bruno, 2008 ; Barrier, 2011; Frances et Lelay, 2012) et d'organisation de l'enseignement supérieur (Musselin, 2001), portent la marque d'une référence croissante à l'univers de l'entreprise. Les relations avec le monde économique contribuent à la définition des valeurs de l'enseignement et de la recherche. Aujourd'hui, elles conduisent à l'intégration de certaines normes professionnelles par les étudiants ou les personnels de l'enseignement supérieur, comme l'esprit d'initiative et la capacité d'adaptation qui participent du «nouvel esprit du capitalisme» décrit par Luc Boltanski et Eve Chiapello (1999).

Dans la littérature académique comme dans le discours syndical et politique (Bongrand, 2012 Bruno 2008 ; Garcia 2009 ; Laval, Vergne, Clément, Dreux 2012), les phénomènes signalés ci-dessus sont souvent regroupés sous la bannière d'une "économi(ci) sation" ou d'une "marchandisation" de l'enseignement supérieur. Ces notions et la diffusion dont elles font l'objet posent plusieurs problèmes.

Tout d'abord, elles tendent à opérer une réduction dommageable en opposant un passé fantasmé à un présent ou un futur dans lesquels les logiques économiques détermineraient entièrement les autres champs d'activité (Bourdieu, 1997). Or, l'ancienneté de l'ancrage économique du système éducatif a été clairement mise en évidence, notamment par J.-M. Chapoulie (2010) selon lequel l'école constitue un moyen de socialisation des forces de travail par l'inculcation d'une discipline. P. Bourdieu et J.-C. Passeron (1970) ont aussi montré la contribution de l'institution scolaire à la reproduction socio-économique par une naturalisation des prétentions statutaires mais aussi les fluctuations de la dynamique historique.

Ensuite, ces concepts incitent à subsumer sous une même catégorie, des phénomènes parfois très différents, et contribuent ainsi à une réification courante qui envisage le

4. Au sens ici de salariés ou travailleurs indépendants du secteur privé ou public (hors secteur éducatif).

5. Actes de la recherche en sciences sociales, "Entreprises académiques ", $\mathrm{n}^{\circ} 148,2003 / 3$.

6. Notamment ceux produits depuis le tournant des années 2000 par les instances européennes et internationales: Processus de Bologne (1999), stratégie de Lisbonne (2000), négociations au sein de l'OMC (Organisation mondiale du commerce) dans le cadre de l'Accord Général sur le Commerce des Services (2000), préconisations de l'OCDE (Organisation de coopération et de développement économiques), etc. 
système éducatif et l'économie comme deux univers circonscrits et distincts, le premier étant menacé par l'expansion du second. Or, une telle représentation perd de vue la pluralité et l'ambiguité des formes que peut prendre la relation entre enseignement supérieur et économie. Ainsi, selon Laval et al. (2012), la nouveauté pour La nouvelle école capitaliste consiste à ne "plus faire illusion" quant à sa fonction reproductive, en préparant plus explicitement les élèves à leur participation future à l'économie marchande. Dans cette perspective, la généralisation des stages en entreprise dans les cursus ou le développement des formations en alternance peuvent être vus comme des vecteurs de socialisation anticipée. La rencontre de l'enseignement supérieur et de l'économie est aussi manifeste dans l'adoption des modes de fonctionnement de l'entreprise privée par les institutions éducatives, ou encore dans l'essor de la spéculation financière sur l'enseignement supérieur, à un niveau international (Vinokur, 2009).

Certes, on peut concevoir que ces phénomènes participent d'un vaste mouvement, aux manifestations plurielles mais doté d'une certaine cohérence. Ainsi, nombre de travailleurs des services dits publics font l'expérience de l'envahissement de leur monde professionnel par des prescriptions jugées économiques, marchandes ou gestionnaires, processus que Frédéric Pierru analyse dans le monde hospitalier comme un " tournant néolibéral" (2012). Il est d'ailleurs salutaire de s'efforcer de saisir le sens qui se dégage de l'apparent désordre du monde, à l'heure où l'on tend plutôt à insister sur l'incommensurable pluralisme des processus sociaux et économiques, renforçant ainsi le morcellement de la connaissance. Cela ne doit toutefois pas conduire à éviter une confrontation empirique à la complexité des relations entre l'enseignement supérieur et le "monde économique " ${ }^{7}$. Dès lors, nous suggérons de penser en termes d'interpénétration entre logiques éducatives et logiques économiques, dans la lignée des travaux de Karl Polanyi (1944) relatifs au désencastrement de l'économie de marché par rapport au social, tout en étant particulièrement attentif aux différentes modalités de ce processus.

Ce dossier entre ainsi dans la fabrique historique et sociale des rapports entre l'enseignement supérieur et le monde économique, sans en négliger les ambivalences. En écho à la journée d'études dont il est issu ${ }^{8}$, il nourrit également une ambition d'ordre

7. A la différence des expressions « monde du travail », « monde de l'entreprise " ou encore « système productif ", la notion de «monde économique " permet de distinguer l'univers composé des entités assurant une production marchande, directement régies par les lois du capitalisme, de l'univers des institutions réalisant une mission dite de service public (comme les établissements d'enseignement supérieur à but non lucratif), mais constituant elles aussi un « monde du travail ». Nous la préférons également à celle de " champ », dans la mesure où les contributions présentées ici ne visent pas à reconstituer précisément la structure des positions et les propriétés spécifiques qui, selon Pierre Bourdieu, caractérisent chacune d'elles et les agents qui les occupent.

8. "Comment traiter des rapports entre enseignement supérieur et "monde économique" ? ", 7 mai 2013, Institut de Recherche Interdisciplinaire en Sciences Sociales (IRISSO), Université Paris Dauphine. 
épistémologique, défendant une approche souple de ces relations, prête aux détours historiques et/ou spatiaux et attentive à la dynamique des médiations.

Non contentes de porter sur des aspects encore mal connus, les contributions réunies dans ce numéro rendent compte de la pluralité des formes prises par la relation considérée, en s'attachant à restituer les configurations dans lesquelles elle se constitue.

Deux articles portent sur l'université et tentent de saisir, avec des entrées différentes, les conditions sociales d'une inflexion des relations de cette institution avec le monde économique. Se plaçant au niveau de la fabrique de la politique universitaire, Christelle Dormoy-Rajramanan relate la construction historique de l'injonction à " ouvrir l'Université au monde extérieur ". Elle montre que la construction de ce mot d'ordre politique est au principe de l'émergence d'une nouvelle représentation de l'enseignement supérieur, plus consensuelle dans son principe que dans ses implications réelles. Suivant une approche plus organisationnelle, Simon Paye décrit, quant à lui, l'adoption de techniques de gestion des ressources humaines (GRH) issues des entreprises privées, par les universités britanniques. Il révèle les conditions sociales de l'essor de la GRH en milieu académique, depuis les années 1970, les modalités de sa mise en place ainsi que ses effets sur la profession académique.

Les deux autres textes s'intéressent à des segments de l'enseignement supérieur réputés plus en prise avec le monde économique : les écoles d'ingénieurs et l'enseignement supérieur privé. Ils montrent pourtant à quel point cette relation ne va pas de soi, qu'elle est toujours le produit d'une construction sociale et se nourrit de la "porosité entre secteur public et secteur privé ", pour reprendre une expression d'Aurélien Casta. Selon lui, l'enseignement privé à but lucratif doit précisément son développement à des dispositifs législatifs. Il décrit en effet les usages que ses promoteurs font, en France, des procédures publiques de reconnaissance et de financement de l'enseignement supérieur. Quant aux écoles d'ingénieurs, Antoine Derouet souligne à quel point leur proximité avec l'entreprise n'est pas une évidence. Il étudie les transformations des relations qu'elles entretiennent au prisme de l'évolution des «enseignements socio-économiques que les écoles intègrent dans leurs cursus".

Ce dossier entend moins porter un regard exhaustif ou synthétique sur la thématique considérée, que traiter de questions circonscrites, en prêtant attention à ce qu'elles révèlent de la dynamique de la relation entre enseignement supérieur et monde économique.

D'une part, il s'agit de montrer l'intérêt d'un décentrement du regard, par une démarche historique ou par l'étude de contextes nationaux différents. Antoine Derouet et Christelle Dormoy-Rajramanan restituent l'historicité et les contingences de la " professionnalisation " des formations supérieures. Avec le premier, on peut suivre, entre 1916 et les années 1970, les différentes configurations liant les écoles d'ingénieurs, les organisations d'employeurs ou de professionnels et l'Etat, et les variations correspon- 
dantes de la définition du «bon enseignant » et de la bonne relation de ces institutions avec l'économie. La seconde montre le caractère stratégique, pour le pouvoir politique confronté à l'agitation de la fin des années 1960, d'une professionnalisation des cursus visant à remettre de l'ordre dans l'institution universitaire en pleine croissance. Simon Paye, quant à lui, tourne son regard vers le Royaume-Uni. La comparaison avec la situation française présente un intérêt non seulement eu égard aux différences de modes de régulation des corps professionnels académiques mais aussi parce que les universités britanniques constituent un laboratoire potentiel de futures réformes en France. Par le détour qu'ils opèrent, ces textes permettent de saisir plus finement les contraintes et les incertitudes qui caractérisent l'enseignement supérieur et contribuent à la recomposition des hiérarchies sociales qui s’y déroule.

D'autre part, dans une intention commune de saisir le mouvement des rapports entre enseignement supérieur et monde économique, les textes insistent sur le travail des intermédiaires'. Ces derniers contribuent en effet à construire l'existence même de ces deux mondes et les modalités particulières de leur mise en relation. Pour ce faire, les perspectives adoptées sont variées. Simon Paye suit la diffusion des dispositifs de gestion, assurée par certains passeurs de ces instruments, issus du monde académique mais aussi - là réside la nouveauté - d'entreprises et d'administrations où ils ont fait leurs armes en tant que professionnels des ressources humaines. Dans le cas des écoles d'ingénieurs, constatant une forte dépendance des enseignements socio-économiques aux enseignants eux-mêmes, en raison d'une faible formalisation de ce curriculum, Antoine Derouet montre que les relations des écoles avec le monde économique peuvent dès lors être saisies via l'analyse des parcours des enseignants, entre entreprise et école. Christelle Dormoy, quant à elle, décrit bien comment des conceptions différentes de l'« ouverture " de l'institution universitaire s'articulent autour d'une injonction floue, au gré de la rencontre de leurs porteurs dans les colloques organisés par l'Association d'étude pour l'expansion de la recherche scientifique (AEERS) ou dans la fabrique même de la loi Faure en 1968. Et si Aurélien Casta s'intéresse aussi aux intermédiaires, c'est en tant qu'espaces où s'imbriquent public et privé, et qui sont investis par les écoles à but lucratif afin d'obtenir des financements et bénéficier de sources de légitimation.

En filigrane des quatre textes se dessinent les interdépendances constitutives du système éducatif. En effet, les universités, les écoles d'ingénieurs, les écoles de commerce ou encore les sections de techniciens supérieurs sont en relation les unes avec les autres, dans un cadre national, voire international. En situation de concurrence, elles entretiennent avec le monde économique des rapports différenciés qui influent sur leurs positions respectives ${ }^{10}$. L'essor d'enquêtes empiriquement étoffées sur des segments de

9. L'intérêt d'une telle perspective est notamment défendu par Olivier Nay et Andy Smith (2002).

10. Le supérieur est également en relation avec les autres niveaux du système éducatif qu'il serait pertinent 
l'espace éducatif, telles que celles présentées ici, encourage le développement de travaux qui ne soient pas des seules monographies mais tentent de penser de concert les situations circonscrites et la multiplicité des interdépendances. Ainsi pourrons-nous interroger à nouveaux frais la cohérence historique des phénomènes observés, que nous n'entendons pas réduire à la mécanique d'une "économicisation " de l'enseignement supérieur.

\section{Bibliographie}

Actes de la recherche en sciences sociales, (2003), "Entreprises académiques ", n 148.

Barrier J. (2011), «Financer la recherche, organiser les relations science-industrie : les politiques de financement sur projet en France (1982-2006) ", dans Bezes Ph. et Siné A. (dir.), Gouverner (par) les finances publiques, Paris, Presses de Sciences Po.

Boltanski L. et Chiapello E. (1999), Le Nouvel Esprit du capitalisme, Paris, Gallimard.

Bongrand P. (2012), " La mise en système et l'économicisation de l'enseignement en France au début des années 1950 : la fonctionnalisation d'une institution ", Politix.

Bourdieu P. (1997), « Le champ économique ", Actes de la recherche en sciences sociales, vol. $119, \mathrm{n}^{\circ} 1$, pp. $48-66$.

Bourdieu P., Passeron J.-C. (1970), La Reproduction, Elements pour une théorie du système d'enseignement, Editions de Minuit.

Bruno I. (2008), À vos marques, prêts... cherchez!: La stratégie européenne de Lisbonne, vers un marché de la recherche, Editions du Croquant, Paris.

Chambard O. (2013), «La promotion de l'entrepreneuriat dans l'enseignement supérieur. Les enjeux d'une création lexicale ", Mots, n 102, pp. 103-120.

Chapoulie J.-M. (2010), L'Ecole d'Etat conquiert la France. Deux siècles de politique scolaire, Presses Universitaires de Rennes.

Durkheim E. (1922), Education et sociologie, Paris, Presses universitaires de France, 1968 (1ère édition : 1922).

Frances J. et Lelay S. (dir.) (2012), Dossier "Qui veut la peau de la recherche publique ?", Mouvements, ${ }^{\circ} 71$.

Garcia S. (2009), «Réformes de Bologne et économicisation de l'enseignement supérieur ", Revue du MAUSS, vol. 1, n 33, pp. 154-172.

de prendre en compte. En ce qui concerne les relations entre formation et emploi, voir Maillard (2012). 
Giret J.-F. (2011), De l'enseignement supérieur de masse à l'économie de la connaissance : la valeur des diplômes en question, rapport d'Habilitation à Diriger des Recherches en sciences économiques, université de Bourgogne.

Hugrée C. (2010), L'échappée belle. Parcours scolaires et cheminements professionnels des étudiants d'origine populaire diplômés de l'université (1970-2010), thèse de doctorat en sociologie, université de Nantes.

Laval Ch., Vergne F., Clément P., Dreux G. (2012), La nouvelle école capitaliste, La Découverte.

Maillard F. (2012), Former, certifier, insérer. Effets et paradoxes de l'injonction à la professionnalisation des diplômes, PUR.

Maurice M., Sellier F., Silvestre J.-J. (1982), Politique d'éducation et organisation industrielle en France et en Allemagne : essai d'analyse sociétale, Paris, PUF.

Moreau G. (dir.) (1999), Les Patrons, l'Etat et la Formation, Paris, La Dispute.

Musselin C. (2001), La longue marche des universités françaises, Paris, PUF.

Nay O., Smith A. (dir.) (2002), Les intermédiaires en politique : médiations et jeux d'institutions, Le gouvernement du compromis. Courtiers et généralistes dans l'action politique, Paris, Economica, pp. 47-86.

Pierru F. (2012), « Le mandarin, le gestionnaire et le consultant. Le tournant néolibéral de la politique hospitalière ", Actes de la recherche en sciences sociales, 4, $\mathrm{n}^{\circ} 194$, pp. 32-51.

Polanyi K. (1983 (1944), La Grande Transformation. aux origines politiques et économiques de notre temps, Gallimard.

Poullaouec T. (2010), Le diplôme, arme des faibles. Les familles ouvrières et l'école, Paris, La Dispute.

Ropé F. et Tanguy L. (dir.) (1994), Savoirs et compétences. De l'usage de ces notions dans l'école et l'entreprise, Paris, L'Harmattan.

Vinokur (2009), "Globalisation du capital et enseignement supérieur », colloque international Les universités au temps de la mondialisation/globalisation et de la compétition pour l'excellence, universite Paris 8, 11-13 mai 2009. 